\title{
胞状铝合金凝固过程中固-液两相区的 附加力场"
}

郑明军 ${ }^{* *}$ 何德坪 ${ }^{* * \dagger}$ 戴 戈

(东南大学材料科学与工程系, 南京 210096)

\begin{abstract}
摘要铝合金在泡沫化过程中和纯铝有着相似性, 但在在凝固过程中具有纯铝所没 有的固液两相区, 单向凝固过程中离固相面距离不同固相含量也不同, 因此会产生一 个导致严重收缩现象的附加力场, 采用物理数学模型与实验相结合的方法, 研究了具 有固一夜两相区胞状铝合金凝固过程的规律, 理论与实验结果符合良好. 在合适的生长 阶段采用各向同时冷却的方法则可以解决这种缺陷。采用这种方法制备的胞状铝合金 的压缩屈服应力比胞状纯铝高 $40 \%$ 以上.
\end{abstract}

\section{关键词胞状铝合金 单向凝固 两相区 附加力场}

泡沫铝是一种以孔隙率为特征的轻质多功能材料, 其结构特点为铝基体中均匀分布着大量的 孔洞. 就其孔结构而言有通孔与闭孔之分, 其中通孔结构叫多孔铝, 闭孔结构称为胞状铝 ${ }^{[1]}$.

胞状铝采用少量金属实现了金属材料多功能化, 包括: 轻质 $(\rho<1)$ 、高比强、能量吸收、 阻尼、声、热、电磁屏蔽等，因而成为 21 世纪前沿热点材料之一 ${ }^{[1 \sim 6]}$.

从 1951 年 ${ }^{[7]}$ 以来, 胞状纯铝一直作为研究的重点, 日本 ${ }^{[8]}$ 、中国 ${ }^{[46]}$ 相继成功, 它可获得 高孔隙率 $(P=85 \%$ 91\%), 孔结构均匀无大孔的制品. 近几年, 剑桥大学、哈佛大学与 MIT 合 作, 在结构性能为主的研究领域取得了重大进展 ${ }^{[1,3]}$, 但对制备未作深人研究. 胞状纯铝的强 度不高, 限制了其应用范围. 为满足高技术领域轻质高孔隙率、高比强的要求, 胞状铝合金就 成为新的前沿研究方向. 文献 $[9,10]$ 分别研究了胞状铝硅 $(P<84 \%)$ 和胞状铝镁合金 $(P<82 \%)$ 的制备和性能, 尚未达到轻质的要求. 迄今, 还未有稳定获得高孔隙率、孔结构均匀、高比强、 高成品率胞状铝合金成功的报道.

本文在分析胞状铝合金制备与胞状纯铝制备差异的基础上, 采用物理数学模型与实验相 结合的方法, 研究了具有固-液两相区胞状铝合金凝固过程的规律, 采用合适的凝固控制等方 法, 稳定获得了高孔隙率 $(P=86 \%$ 91\%)、孔结构均匀、无集中大孔、无显著收缩、高比强、 高成品率的胞状铝合金.

\section{1 实验研究方法}

\section{1 实验设备}

实验设备应满足 $\mathrm{Al}$ 合金熔化及泡沫化制备工艺, 包括增黏剂混匀、发泡剂混匀、凝固控

2001-11-28 收稿, 2002-04-05 收修改稿

* 国家自然科学基金重点资助项目(批准号: 50231010)和国家自然科学基金资助项目(批准号: 50081002, 19982001)

** 同等贡献作者

$\dagger$ 联系人, E-mail: dphe@ seu.edu.cn 
制等. 熔体泡沫化制备过程中界面推移测量、采用扫描仪、计算机及专用软件分析孔结构, 详 见文献[4 6].

与胞状纯铝制备相比，设备及原材料有以下差别:

(1) 以铝合金 ZL111 $(8.0 \% \sim 10.0 \% \mathrm{Si}, 1.3 \% \sim 1.8 \% \mathrm{Cu}, 0.4 \% \sim 0.6 \% \mathrm{Mg}, 0.1 \% \sim 0.35 \% \mathrm{Mn}, 0.1 \%$ $0.35 \% \mathrm{Ti}$ ) 作为基体材料, 为了比较亦采用了少量的纯铝.

(2) 坩埚内部尺寸为 $100 \mathrm{~mm} \times 100 \mathrm{~mm} \times 200 \mathrm{~mm}$ 的方形坩埚.

(3) 在泡沫化制备中首次采用了高精度 $\left(1 \times 10^{-3} \mathrm{~N} \cdot \mathrm{m}\right)$ 扭矩仪-计算机黏度测量系统, 以精 确控制制备过程.

(4) 改进了凝固控制设备, 可以单向亦可以多向凝固.

（5）仔细切割了所有样品, 采用扫描仪、计算机孔结构分析系统分析孔结构, 以研究胞状 铝合金凝固方式对孔结构的影响.

为了保证凝固实验中数据的重复性, 共进行了 50 余次实验.

\section{2 研究方法及实验现象}

（ｉ）研究方法现阶段材料研究由于其复杂性多采用实验研究方法, 我们力求采用实 验与理论相结合的方法. 文献[11]采用物理与实验相结合的模拟法, 文献[11,12]采用从数学推 演到物理现象的演绎法, 而文献[4]则采用了从物理现象引导出数学表示的归纳法. 本文采用 了后者. 它对于目前尚以实验为主的材料研究非常重要, 其难点在于抓住复杂实验现象的物 理本质，建立物理模型、采用合适的数学方法获得与实验结果吻合的结果.

(ii ) 实验现象铝合金熔体泡沫的凝固是胞状铝合金制备的一个关键步骤, 凝固过程 的控制不当会引起各种缺陷，甚至 导致试样制备的失败. 胞状纯铝在 制备 $100 \mathrm{~mm} \times 100 \mathrm{~mm} \times 200 \mathrm{~mm}$ 样品时采取单向凝固, 由于凝固是 从一个方向进行，因此未凝固区就 可以补充已凝固区的收缩, 不容易 形成内部空洞, 单向凝固可以减少 由凝固收缩所造成的缺陷. 但是, 对于用同样制备方法获得的铝合 金泡沫进行单向冷却 $30 \mathrm{~s}$ 以后, 胞 状铝合金样品从一定高度 $x$ 在 $5 \mathrm{~s}$ 内会出现层叠状收缩 $(x$ 沿中心纵 轴线测量, 精度为 $0.1 \mathrm{~mm})$, 收缩 量达到 $30 \%$ 以上, 孔也由多面体变 为扁形, 如图 1(a), 而在高度小于 $x$ 的区域, 胞孔则仍为正常多面体. 纯铝基体样品则没发生这种情况, 如图 1(b). 随冷却强度的变化, 收 缩的程度也有所不同, 严重时, 会
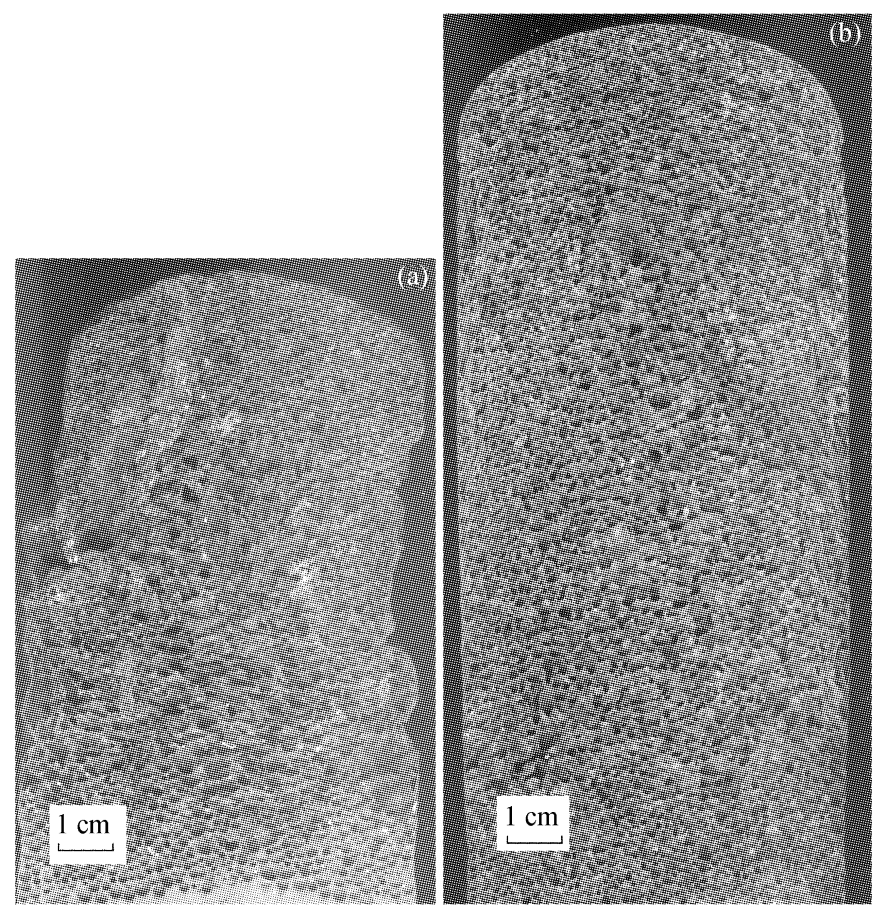

图 1 单向凝固下胞状铝合金及胞状纯铝样品 (a) 胞状铝合金试样的收缩; (b) 胞状纯铝 
使实验完全失败. 为了制备高孔隙率、高比强胞状 $\mathrm{Al}$ 合金, 必须揭示这一实验现象背后的物 理本质，分析引起收缩的驱动力，以便找到解决这个问题的途径.

\section{2 实验结果及分析}

$\mathrm{Al}$ 合金及纯铝的泡沫形成过程分为熔体泡沫生长及凝固过程, 两者熔体中的生长过程具 有相似性，而差异在于凝固过程.

\section{1 纯铝及铝合金熔体泡沫生长过程的相似性}

图 2 是在某一固定工艺条件下的熔体泡沫的生长曲线(未经冷却), 纯铝熔体和 $\mathrm{Al}$ 合金熔

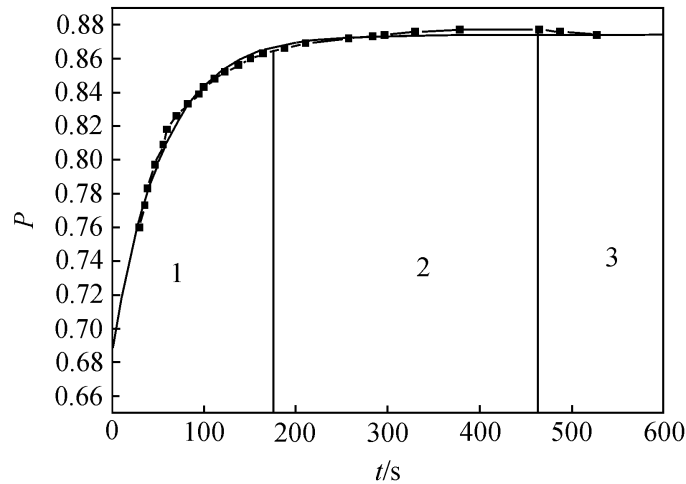

图 $2 \mathrm{Al}$ 合金与 $\mathrm{Al}$ 熔体泡沫的生长曲线 体的生长曲线类似(测量方法见文献[4]), 泡沫 生长过程都可以分为 3 个阶段: 快速长大阶段、 稳定阶段和胞体失稳阶段.

第 1 阶段，当发泡剂加人高温熔体内时，大 量的气体放出, 气泡形核并长大, 胞体由球形 向多面体转变, 这一阶段时间较短 $(<180 \mathrm{~s})$, 生 长速度很快. 影响这一阶段的主要因素包括熔 体黏度、发泡剂用量、发泡温度、发泡搅拌时 间等 ${ }^{1)}$. 这些因素通过改变气液之间的物理传 递性能和气泡膨胀的热动力来影响胞体的膨胀, 并且相互之间也会产生影响，情况比较复杂， 胞体内部是一个非力学平衡体系, 各气泡在不停地运动和膨胀.

第 2 阶段大致在 180 450 s 之间, 熔体泡沫孔隙率 $P$ 在这一阶段基本不再变化, 胞体完全 转变为稳定的近似多面体结构(面的交界处为 Gibbs-Plateau 通道, 为曲面), 此时各气泡基本形 成一个力学平衡结构, 气泡保持稳定, 此时是凝固的最佳时机. 本研究的凝固从该阶段开始. 此 时如不开始冷却, 胞体生长将进入第 3 阶段.

第 3 阶段, 胞体失稳, 气泡开始破裂合并. 因而如在第 3 阶段凝固就无法获得均匀的孔结构.

\section{$2.2 \mathrm{Al}$ 合金及纯铝泡沫在凝固过程中的差异及铝合金泡沫收缩驱动力模型}

为了分析 $\mathrm{Al}$ 合金泡沫在单向凝固过程引起向下显著收缩的驱动力，作如下假设:

(i) 凝固前 $\mathrm{Al}$ 和 $\mathrm{Al}$ 合金熔体内温度恒定均匀;

(ii) 在充分混匀条件下, 熔体中的气泡均匀;

(iii) 从泡沫熔体生长过程的稳定阶段开始冷却;

(iv) 泡沫从稳定阶段开始到凝固结束, 胞的总数不变.

从第 2 阶段开始单向凝固以后, 铝合金熔体泡沫开始出现不同于纯铝泡沫的收缩特性, 这 一现象表明, 纯铝泡沫在单向凝固过程中, 虽然存在凝固收缩力, 但是由于凝固收缩很小(纯 铝及其合金的线收缩率在 1\%左右), 所以仍能近似保持体系的力学平衡, 不会发生图 1(a)所示 的收缩现象. 而铝合金泡沫在单向凝固过程中显然存在一个改变体系内力学平衡状态的驱动 力 $F$ (图 3), 这个驱动力在纯铝泡沫中是不存在的, 可以判断, 这一差异与两种基体的物理性质 不同有关. 铝合金泡沫与纯铝泡沫凝固的一个明显区别是，纯铝泡沫有一个确定的凝固点，而 
铝合金泡沫的凝固则是在一定温度范围(ZL111 的液 相线和固相线分别为 $596^{\circ} \mathrm{C}$ 和 $538^{\circ} \mathrm{C}$ )内. 冷却进行 到时间 $\tau$ 时, 纯铝泡沫和铝合金泡沫体内部都会 产生一个温度场, 从下至上温度升高, 但是纯铝泡 沫只有一个固液界面, 而铝合金泡沫则会在一定 高度 $\varepsilon$ 会出现一个液、固相共存的区域 B(图 3), $\varepsilon$ 随冷却时间的增加而增大. 在 B 区域中, 胞壁由液固两相组成, 随高度 $x$ 的不同液、固相含量的比例 也不同, 高度越小所含固相越多. 而纯铝泡沫在单 向凝固过程中虽然也会形成温度场, 但是它只有 一个固液界面, 不存在两相区.

由于固相原子之间的距离较液相小, 因此固 相原子之间的相互作用力较液相大，表现在表面 现象上, 就是固相的表面张力较液相大. 文献[13] 给出了固相金属表面张力的近似算法:

$$
\sigma=\frac{1}{2}\left(56400\left(\frac{\rho}{Z}\right)^{4 / 3}\right) \times 10^{-7} \mathrm{~J} \cdot \mathrm{cm}^{-2}
$$

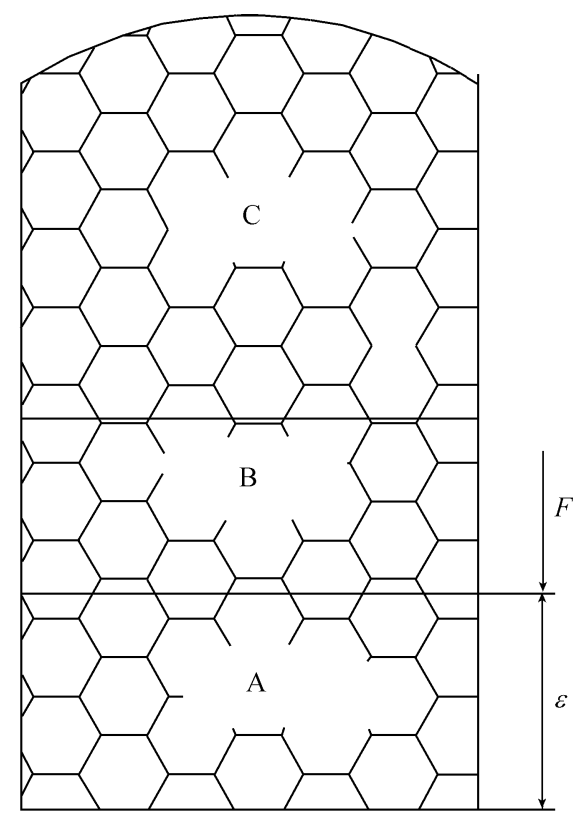

图 3 单向凝固过程中的 3 个区域 $\mathrm{A}$ 固相, $\mathrm{B}$ 固相 + 液相, C 液相

$\rho$ 为金属密度, $\mathrm{g} / \mathrm{cm}^{3}, Z$ 为原子序数.

将铝的参数代人(1)式并换算成国际标准单位, 可以得到固相的表面张力为 $3.47 \mathrm{~J} / \mathrm{m}^{2}$, 而 铝熔液的表面张力 ${ }^{[14]}$ 为 $0.86 \mathrm{~J} / \mathrm{m}^{2}$, 仅为固相表面张力的 $1 / 4$, 这样在 $\mathrm{B}$ 区内将出现一个表面张 力梯度 $\frac{\partial \sigma}{\partial x}$ (图 4), 这个力场由液相的表面张力、固相的表面张力以及液相线和固相线的形状所 确定, 液相和固相的表面张力相差越大, 液相面和固相面的距离越近, 这个张力场也就越大. 这样在 $\mathrm{Al}$ 合金泡沫熔体中, 原本如纯铝泡沫熔体内的力学平衡在单向凝固过程中将被破坏.

从单个胞进行考虑, 由于 $\mathrm{B}$ 区胞壁从由液相构成转变为由液固两相构成, 因此表面张力将增 大 $\Delta \sigma$ ( $\Delta \sigma$ 和固相含量相关), 并引起作用在胞壁及 Gibbs-Plateau 通道上的附加压力从液相的

$$
\Delta p_{1}=\frac{2 \sigma_{l}}{r},
$$

$r$ : 胞壁的曲率半径, 从而增大为 $\Delta p_{2}=\frac{2\left(\sigma_{l}+\Delta \sigma\right)}{r}$, 这个附加压力在固液界面端约增大到液态 时附加压力的 4 倍, 在 B 区远固液端则和液态时附加压力相同, 那么 B 区胞内原本的气压不 足以承受这个附加压力的增大，导致胞体的收缩.

取 B 区中一层胞来说明, 如图 5 所示, 各胞在沿凝固方向上在附加压力的作用下存在一个 指向胞体内部的力 $\Delta p_{2}$, 而横截面方向则由于熔体和坩埚壁结合紧密, 有一定的黏结力 $F$, 不 易分离, 只要这个结合力比 $\Delta p_{2}$ 大, 横向部分就不会收缩; 上部因为是液态, 不能支持附加压 力的增加, 于是气泡就向下收缩, 如果得到足够的响应时间, 即收缩速度增加到足够大, 胞体 则会出现图 1(b)所示的胞孔变扁的情况, 而在这一响应时间以前的已凝固部分由于收缩速度 很小, 变形不大, 所以可以近似保持原来的形状. 固相面推移速度与冷却强度有关, 因此收缩 


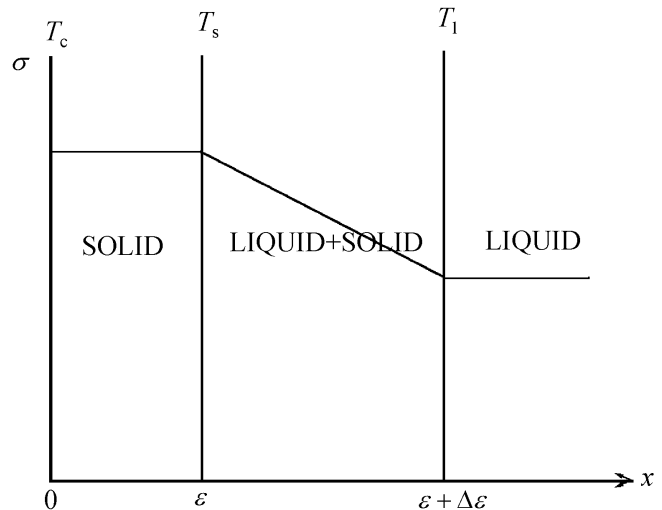

图 4 凝固方向和表面张力

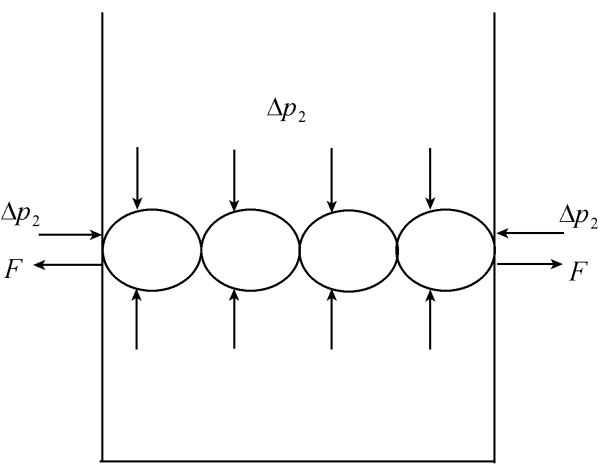

图 5 B 区胞体受力示意图

开始层所在的位置 $(x)$ 与冷却强度相关, 冷却强度越大, 出现胞孔变扁的位置离冷却面越远. 取 $\mathrm{B}$ 区下端层面段考虑, 在不同冷却强度下, 这一段的附加压力可以认为近似相等(都约为液 态时的 4 倍), 从力学原理可知, 收缩的加速度也就相同, 那么达到明显收缩(收缩量 $3 \%$ )时的

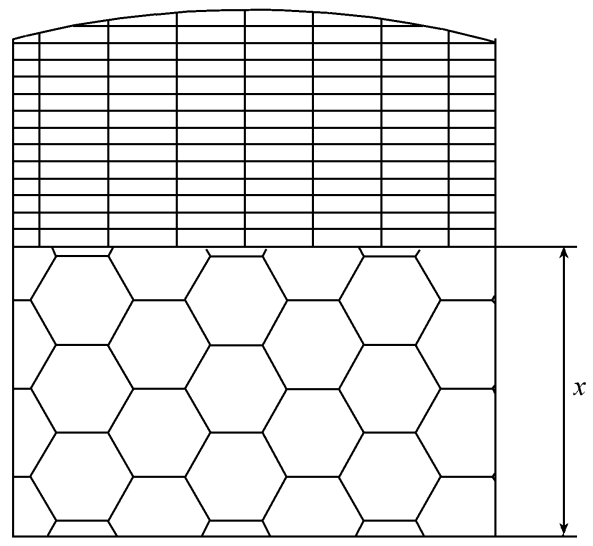

图 6 冷却面和收缩开始面的距离

时间相同, 即响应时间应与冷却强度无关. 在本实验 条件下, 经实验测定, 不同冷却强度下, 单向凝固开 始 $30 \mathrm{~s}$ 后 $\mathrm{Al}$ 合金泡沫熔体总会开始出现明显收缩(本 实验中定义沿高度收缩量达到 $3 \%$ 时定义为明显收缩 开始). 一旦明显收缩开始, 收缩速度就会越来越快.

通过凝固过程的传热学分析 ${ }^{[15]}$ 并考虑泡沫的 存在 ${ }^{1)}$, 可以计算获得发生明显收缩时(冷却开始后 $30 \mathrm{~s}$ )固相面所在的位置, 也就是 $\mathrm{Al}$ 合金泡沫熔体开 始明显收缩的面和冷却面(底面)的距离 $x$ (图 6), 如 果计算出来的 $30 \mathrm{~s}$ 时固相面的位置和实验中测量 所得的明显收缩开始面所在位置相同, 就可以验证 这一模型的正确性.

\section{3 传热学分析}

为简化分析，进行如下假设：

(1) 固相部分和凝固区域的物理性能与温度无关, 但互相之间可能不同.

(2) 温度仅沿 $x$ 轴变化.

(3) 热传导是惟一的传热方式.

（4）导热率随孔隙率的变化忽略不计(因为孔隙率在稳定阶段的变化很小).

(5) 熔化热是惟一的内热来源.

(6) 固相分数与距离的关系为线性, 在液相线为 0 , 在固相线为 $f_{s u}$. 假设(6)可以写为

$$
f_{s}=f_{s u}(1-\bar{x})
$$

1) 戴 戈. Al-Si 合金泡沫形成过程中黏度测量及温场数值模拟探索. 东南大学硕士学位论文, 2001. 41 61 
其中, $\bar{x}$ 无量纲可变参数, 定义为 $\frac{x-\varepsilon}{\Delta \varepsilon}, \varepsilon$ 已凝固部分厚度, $\mathrm{m} ; \Delta \varepsilon$ 正在凝固区域的厚度, $\mathrm{m}$; 当 凝固区域凝固的时候, 凝固潜热相应的释放, 可作为热源处理, 表达为

$$
Q=\rho L \frac{\mathrm{d} f_{s}}{\mathrm{~d} t},
$$

式中, $\rho$ 为铝合金泡沫的密度, $\mathrm{kg} / \mathrm{m}^{3}, L$ 为凝固潜热, $\mathrm{J} / \mathrm{kg}$.

结合(3), (4)式

$$
Q=\frac{\rho L f_{s u}}{\Delta \varepsilon}\left(\frac{\mathrm{d} \varepsilon}{\mathrm{d} t}+\bar{x} \frac{\mathrm{d} \Delta \varepsilon}{\mathrm{d} t}\right) .
$$

Fourier 传热学公式用在已凝固区域和正在凝固区域, 结合适当的初始条件和边界条件就 可以解出铝合金泡沫凝固时的温场分布, 完整的数学表达式为

$$
\begin{gathered}
\frac{\partial^{2} T_{1}}{\partial x^{2}}=\frac{1}{\alpha_{1}} \frac{\partial T_{1}}{\partial t}, \\
\frac{\partial^{2} T_{2}}{\partial x^{2}}+\frac{Q}{\lambda_{2}}=\frac{1}{\alpha_{2}} \frac{\partial T_{2}}{\partial t} .
\end{gathered}
$$

边界条件为

(1) $T_{1}(x=0)=-\left(T_{s}-T_{c}\right)$,

(2) $T_{1}(x=\varepsilon)=0$

(3) $T_{2}(x=\varepsilon)=0$,

(4) $T_{2}(x=\varepsilon+\Delta \varepsilon)=T_{l}-T_{s}=\Delta T$,

(5) $\frac{\partial T_{2}}{\partial x}(x=\varepsilon+\Delta \varepsilon)=0$,

(6) $\lambda_{1} \frac{\partial T_{1}}{\partial x}(x=\varepsilon)=\lambda_{2} \frac{\partial T_{2}}{\partial x}(x=\varepsilon)+\rho L\left(1-f_{s u}\right) \frac{\mathrm{d} \varepsilon}{\mathrm{d} t}$;

初始条件为

(1) $\mathcal{E}(t=0)=0$,

(2) $\Delta \varepsilon(\dot{t}=0)=0$,

其中, $\alpha_{1}$ =固相热扩散率, $\mathrm{m}^{2} \cdot \mathrm{s}^{-1} ; \alpha=\frac{\lambda}{\rho c}, c$ 为材料的热容, $\mathrm{J} / \mathrm{kg} \cdot \mathrm{K} ; \alpha_{2}=$ 液相热扩散率, $\mathrm{m}^{2} \cdot \mathrm{s}^{-1} ; T_{1}=$ 固相部分温度, $\mathrm{K} ; T_{2}=$ 液相部分温度, $\mathrm{K} ; T_{l}=$ 液相线温度, $\mathrm{K} ; T_{\mathrm{c}}=$ 冷却表面温度, $\mathrm{K}$; $\varepsilon=$ 已凝固部分厚度, $\mathrm{m} ; \Delta \varepsilon=$ 液固相共存区域, $\mathrm{m} ; \lambda_{1}=$ 固相导热系数, $\mathrm{w} \cdot(\mathrm{m} \cdot \mathrm{K})^{-1} ; \lambda_{2}=$ 液 相导热系数, $\mathrm{w} \cdot(\mathrm{m} \cdot \mathrm{K})^{-1}$.

\section{4 理论计算与实验结果比较}

由于胞状铝合金的导热系数主要由孔隙率决定, 固、液态的孔隙率基本相同, 所以本文取

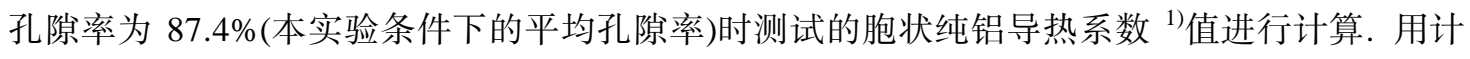
算机对(6), (7)所示的方程进行数值求解 ${ }^{2)}$, 可以获得在某一冷却强度条件下开始收缩的固相面

1) 吴照金, 铝合金泡沫化和凝固过程中孔结构的变化规律及其控制. 东南大学博士学位论文, 2000. 55

2) 见 474 页脚注 1) 
位置. 计算机数值计算中的有关热物性见表 1.

表 1 坩埚和胞状铝合金的物理参数

\begin{tabular}{lcc}
\hline & 坩埚 & 合金泡沫 \\
\hline$\rho / \mathrm{kg} \cdot \mathrm{m}^{-3}$ & $7.86 \times 10^{3}$ & $3.51 \times 10^{2}(P=87 \%)$ \\
$\lambda / \mathrm{w} \cdot(\mathrm{m} \cdot \mathrm{K})^{-1}$ & 46.89 & $0.1297^{1)}$ \\
$L / \mathrm{J} \cdot \mathrm{kg}^{-1}$ & - & $3.96 \times 10^{5}$ \\
$T s / \mathrm{K}$ & - & 811 \\
$c / \mathrm{J} \cdot(\mathrm{kg} \cdot \mathrm{K})^{-1}$ & $5.78 \times 10^{2}$ & 0.126 \\
$T_{l} / \mathrm{K}$ & - & 869 \\
\hline
\end{tabular}

计算结果和实验数值列表如下:

\begin{tabular}{ccc}
\hline 散热系数 $w / \mathrm{m}^{2} \cdot \mathrm{K}^{-1}$ & \multicolumn{2}{c}{$x / \mathrm{mm}$} \\
\cline { 2 - 3 } & 理论计算值 & 实验测定值 \\
\hline 2075 & 19.8 & 21.0 \\
2729 & 22.5 & 24.2 \\
3031 & 30.4 & 33.5 \\
\hline
\end{tabular}
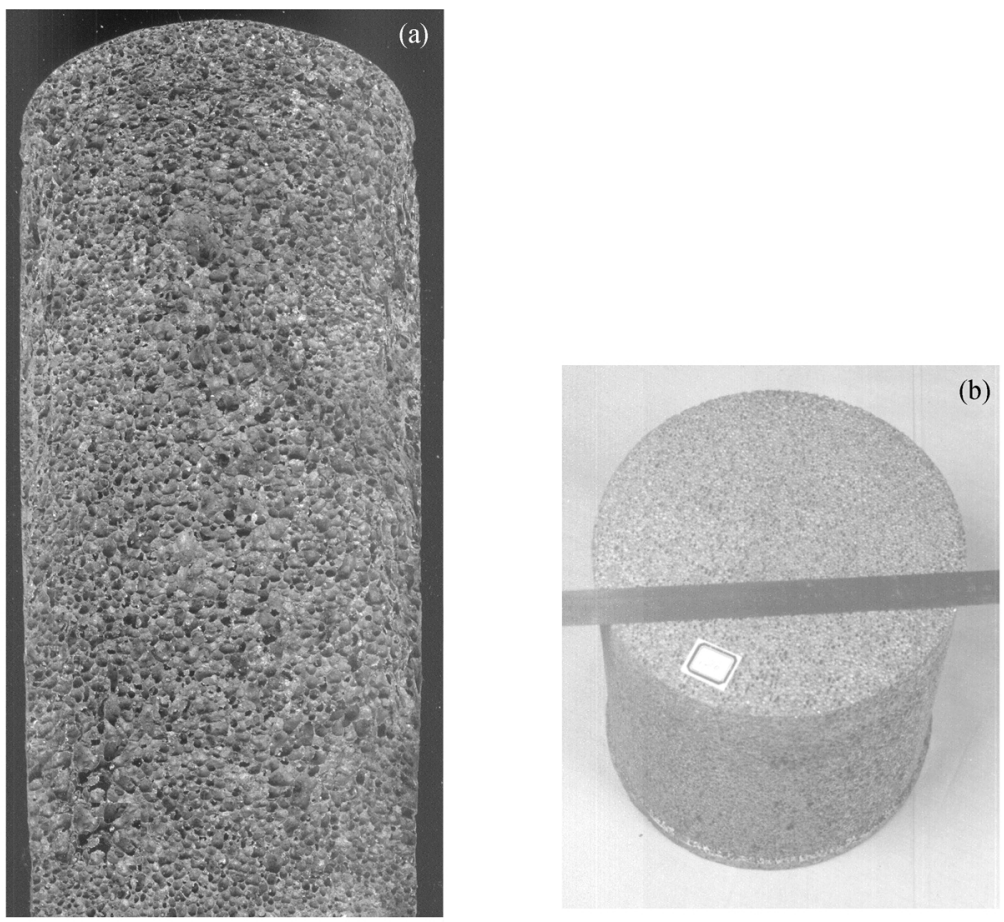

图 7 胞状铝合金样品

(a) $100 \mathrm{~mm} \times 100 \mathrm{~mm}$; (b) $\phi 260 \mathrm{~mm}$

计算中取了 3 个散热系数(即 3 种冷却强度), 可以看到, 实验结果和计算结果较为接近. 由于模型中假设单向导热, 而实际上, 由于坩埚壁面也会少量散热, 并且最靠近固相面的部分 
没有足够的响应时间, 不会有明显的收缩, 因 此实验结果要比理论计算值大.

\section{3 讨论}

\section{1 解决铝合金泡沫凝固过程迅速收缩的途} 径

分析了 $\mathrm{Al}$ 合金泡沫在单向凝固过程中由 于固液二相区附加力场所造成的收缩, 就可以 找到解决这一难题的新途径. 由于铝合金和纯 铝的不同凝固特性, 胞状铝合金的凝固不能采 用单向凝固, 因此必须考虑整体凝固. 整体凝 固时虽然还是存在表面张力梯度的问题, 但是 由于样品外层已经凝固, 可以提供足够的支撑

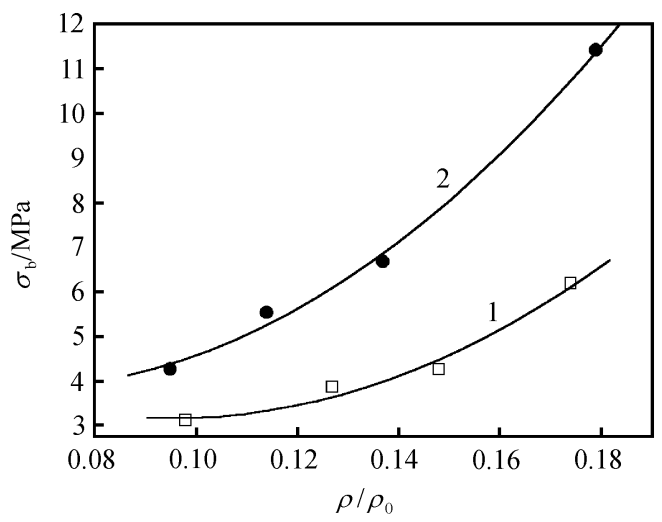

图 8 胞状纯铝和胞状铝合金的压缩屈服应力 1 示胞状纯铝; 2 示胞状铝合金 力以抵消附加压力差的作用, 可以完全解决 $\mathrm{Al}$ 合金泡沫这一特有的凝固收缩问题.

\section{2 采取措施后获得的制品及性能}

图 7(a), (b)分别是采用正确的冷却方法制备的 $100 \mathrm{~mm} \times 100 \mathrm{~mm} \times 200 \mathrm{~mm}$ 和 $\phi 260 \mathrm{~mm} \times$ $300 \mathrm{~mm}$ 的样品, 可以看到样品具有高孔隙率 $(P=86 \%$ 91\%)、孔结构均匀、高成品率 $(>90 \%)$ 、 无图 1(a)所示的层叠状收缩缺陷. 图 8 是胞状铝合金和胞状纯铝压缩屈服应力的比较, 可以看 到，胞状铝合金的屈服应力比胞状纯铝至少高 $40 \%$, 性能得到了大幅提升.

\section{4 结论}

（i ）胞状纯铝和胞状铝合金各自熔体泡沫的生长具有相似性.

(ii ) 在单向凝固过程中胞状铝合金由于具有固液两相区，在两相区会产生一个附加力场, 导致在距冷却面一定高度产生层叠状收缩.

(iii) 采用物理模型与实验相结合的方法研究了具有固-液两相区的胞状 $\mathrm{Al}$ 合金凝固规律, 理论与实验结果吻合良好.

(iv) 采用正确的冷却凝固方法制备的胞状铝合金可以消除特有的层叠状收缩缺陷, 获得 孔隙率高度均匀的轻质、高比强胞状铝合金, 其压缩屈服应力比胞状纯铝高 $40 \%$ 以上.

\section{参考文献}

1 Ashby M F, Evans A G, et al. Metal Foams: A Design Guide. Boston: B-H Press, 2000. 1 5

2 John B, Ashby M F, Fleck N A. Cellular Metals and Metal Foaming Technology. In: International Conference on Cellular Metals and Metal Foaming Technology. Bremen, 2001. Bremen: Verlag MIT, 2001. 5 55

3 Gibson L J, Ashby M F. Cellular Solids-Structure and Properties. Second Edition. Great Britain: A Wheaton \& Co Ltd Exeter, 1999. $1 \sim 10$

4 吴照金, 何德坪. 泡沫铝凝固过程中孔隙率的变化. 科学通报, 2000, 45(8): 829 835

5 吴照金, 何德坪. 泡沫 Al 孔结构的影响因素. 材料研究学报, 2000, 14(3): 277 282

6 宋振伦, 何德坪. Al 熔体泡沫形成过程中黏度对孔结构的影响. 材料研究学报, 1997, 11(3): 275 279

7 Elliott J C. US Patent, 2751289, 1956 
8 Ueno H, Akiyama S. Effects of calcium addition on the foamability of molten aluminum. Light Metal (in Japan), 1987, 37(1): $42 \sim 47$

9 王 斌, 何德坪, 舒光冀. 泡沫铝合金的压缩性能及其能量吸收. 金属学报, 2000, 36(10): 1037 1040

10 Fusheng H, Zhenggang Z. The mechanical behavior of foamed aluminum. Journal of Materials Science, 1999, 34: 291 299

11 何德坪, 闻德蒜, 张 勇, 等. 铝熔体在多孔介质中的渗流过程. 材料研究学报, 1997, 11(2): 113 119

12 杨东辉, 何德坪. 多孔铝合金的孔隙率. 中国科学, B 辑, 2001, 31(3): 265 271

13 亚当森 A W. 表面的物理化学. 北京: 科学出版社, 1986. 269

14 李庆春. 铸件形成理论基础. 北京: 机械工业出版社, 1982. 22

15 Tien R H, Geiger G E. A heat-transfer analysis of the solidification of a binary eutectic system. Transactions of the ASME, 1967(8): 230 234 